\title{
Effects of ankle fatigue on functional reflex activity during gait perturbations in young and elderly men
}

\author{
Urs Granacher ${ }^{a, *}$, Markus Gruber ${ }^{b, * *}$, Dominik Förderer ${ }^{c}$, Dieter Strass ${ }^{c}$, Albert Gollhofer ${ }^{c}$ \\ "Instinte of Exercise and Heaith Sciences, University of Base!. St. Jakob-Tum. Birsstrasse 3208. 4052 Basel. Switzerland \\ Deparment of Training and Movement Science. University of Potsdam. Am Neuen Palais 10. 14469 Polsdan. Cermany \\ Institute of Sport and Sport Science, University of Freiburg, Freiburg, Germany
}

\section{Keywords:}

Seniors

Aging

Postural contro

Neuromuscular latigue

lsokinetic fatigue protocol

\begin{abstract}
There is growing evidence that aging and muscle fatigue tesult in impaired postural reflexes in humans. Therefore, the objective of this study was to examine the effects of ankle latigue on functional reflex activity (FRA) during gait perturbations in young and elderiy men.

Twenty-eight young $(27.0 \div 3.1$ years, $n=14)$ and old $(67.2 \div 3.7$ years, $n=14)$ healthy active men participated in this study. Fatigue of the plantafflexors and dorsiflexors was induced by isokinetic contractions. Pre and post-fatigue, subjects were tested for their ability to compensate for decelerating gait perturbations while walking on a treadmill. Latency, FRA of lower extremity muscles and angular velocity of the ankle joint complex were analysed by means of surface electromyography and goniometry.

After the fatigue protocol, no significant main and interaction effects were detected for the parameter latency in $\mathrm{m}$. tibialis anterior (TA). For both groups, a significant pre to post-test decrease in FRA in TA $(p<.001)$ was observed coming along with increases in antagonist coactivity ( $p=.013)$ and maximal angular velocity of the ankle joint $(p=.007)$. However, no significant group $\times$ test interactions were found for the three parameters.

Ankle fatigue has an impact on the ability to compensate for gait perturbations in young and elderly adults. However, no significant differences in all analysed parameters were decected between young and elderly subjects. These resuits may imply that age-related deteriorations in the postural control system do not specifically affect the ability to compensate for gait perturbations under fatigued condition.
\end{abstract}

\section{Introduction}

The number of senior citizens aged 65 and older has substantially increased in societies of western industrial countries. A serious problem of the aging population is the susceptibility of sustaining a fall. Twenty-eight to $35 \%$ of individuals over the age of 65 years experience at least one fall over a 1 -year period [1]. Furthermore, it was reported that slips and trips account for 30 $50 \%$ of falls in community-dweiling older adults [2]. Numerous epidemiological studies have identified a multitude of risk factors for falling. In fact, deficits in postural control substantially contribute to the increased fall incidence in seniors [3]. Agerelated degenerative changes in muscle mass, sensory function,

\footnotetext{
* Corresponding author. Tel.: +410613778735 ; fax: +410613778758 .

* Corresponding author. Tel.: $+4903319771120 ;$ fax: +4903319771263. E-mail adidresses: urs.granacher@unibas.ch (U. Granacher), markus gruberoun-potsdan.de (M. Gruber).
}

and/or neural processing may impede the ability to control posture effectively [4].

There is evidence in literature that balance impairments, greater stride-to-stride variability, the inhibition of gait quality and thus the increased occurrence of falls is not oniy caused by biologic aging but also by fatigue of the lower leg muscles $[5,6]$. Parijat and Lockhart [6] observed that localised muscle fatigue of the quadriceps affected various kinematic and kinetic gait parameters that are linked with a higher ris of slip-induced falls in young healthy adults. Helbostad et al. [5] reported that a repeated sit-co-stand task affected gait control in older persons in terms of an increased variability in step width and length.

From a functional point of view, it is of interest to know, whether muscle fatigue has similar or different effects on dynamic stability control during postural perturbations in young and elderly subjects. Recently, Mademli et al. [7] investigated the effects of muscle fatigue on the dynamic stability control of young and older adults after inducing a sudden perturbation from a fixed 
Table 1

Characteristics of the study cohort.

\begin{tabular}{lcc}
\hline Characteristic & Young men, $n=14$ & Elderly men, $n=14$ \\
\hline Age (years) & $27.0=3.1$ & $67.2=3.7$ \\
Body height $(\mathrm{cm})$ & $178.4=6.5$ & $175.0 \pm 4.8$ \\
Body mass $(\mathrm{kg})$ & $74.5 \pm 6.3$ & $77.0 \pm 5.5$ \\
Body mass index $\left(\mathrm{kg} / \mathrm{m}^{2}\right)$ & $23.5 \pm 0.8$ & $25.1=2.1$ \\
Acrivity level $($ h $/$ week & $13 \pm 8$ & $13 \pm 3$
\end{tabular}

Note: Vaiues are mean 5 SD.

forward inclined position. The authors were able to demonstrate a lower margin of stability (boundary of the base of support) at touchdown after the forward fall in old compared to young adults. In addition, muscle fatigue of the knee extensors and flexors did not influence the margins of stability in both experimental groups [7].

Since chances of sustaining a fall are particularly high during slipping or tripping situations in fatigued condition $[2,6]$, the objective of this study was to examine the effects of ankle fatigue, as established by standard criteria, on functional reflex activity (FRA) ${ }^{1}$ during gait perturbations in young and elderly men. We hypothesized that fatigue of the muscles encompassing the ankle joint would decrease FRA of muscles compensating for gait perturbations in young and elderly men, with the elderly subjects showing larger decreases than the young.

\section{Methods}

\subsection{Study popularion}

Fourteen young and fourteen elderly men with no statistically significant anthropometrical differences in body mass, body height, and BMI provided writter informed consent to participate in the study after experimental procedures were explained (Table 1). The subjects were healthy with no previous lower extremity trauma and no history of serious muscular, neurological, cardiovascular, metabolic and inflammatory diseases. Before the tests of the study started, participants were asked to complete the validated "Freiburg questionnaire for everyday and sports activities $[10]$. The young and elderiy subjects were classined as pinysically acive. According to the lateral preference inventory $[11\}$, all subjects were right leg dominant. All experiments were conducred according to the larest revision of the declaration of Heisinki.

\subsection{Testing protocol}

in order to analyse effects of ankle farigue on FRA during gait perturbations in young and elderty men, all subiects were tested for treadmill percurbation impulses. Thereafter, they performed an ankle fatigue protocol on an isokinetic device and were subsequently re-tested for treadmill perturbation impulses.

\subsubsection{Treadmill perturbation}

Before the actual resing started, subjects were provided with standardized lowcut athletic footwear (Adidas". Spezial, Herzogenaurach, Germany) to minimize variability and to control for shoe type and design. Thereafrer, parricipants were accustomed to walking on a treadmill for a 5 min wam-up period. Decelerating perturbation impuises were randomly induced at distinct phases of the gain cycie while subjects were walking on a treadmill (Woodway" Weil am Rhein, Germany) in this way, an everyday stumbling situation was transferred into a laboratory condition. The application of decelerating perturbation impulses at heel strike while walking on a treadmill induces an everyday stumbling stimulus which moves the body's center of mass closer to the edge of the base of suppont. As a reactive response, the prime mover TA is activated to bring the body's cencer of mass back over the base of support [12]. For safety reasons, subjects wore a chest harness systen. The treadmill was powered by a DC motor with a gear unit, a tachomete generator, and a transistor - servo-amplifier in a foum-quadrant system. The impulse capacity of the amplifier (MTR-7025) produced $12.750 \mathrm{~W}$ for $1 \mathrm{~s}$. This allowed rapid decelerating impulses of the readnill from $3.5 \mathrm{~km} / \mathrm{h}$ to a backward velocity of $0.6 \mathrm{~km} / \mathrm{h}$ within $0.4 \mathrm{~s}$. Therefore, the readmill was not only decelerared to zero but the direction of the bell movement was also reversed. Elecrical switches implemented into inlays of the right shoe (underneath the heel and the ball of

FRA refers to reflexive (involuntary) activations of muscles of the lower extremities for the purpose of compensating for gait perturbations while walking on a treadmill) $\{8.9\}$

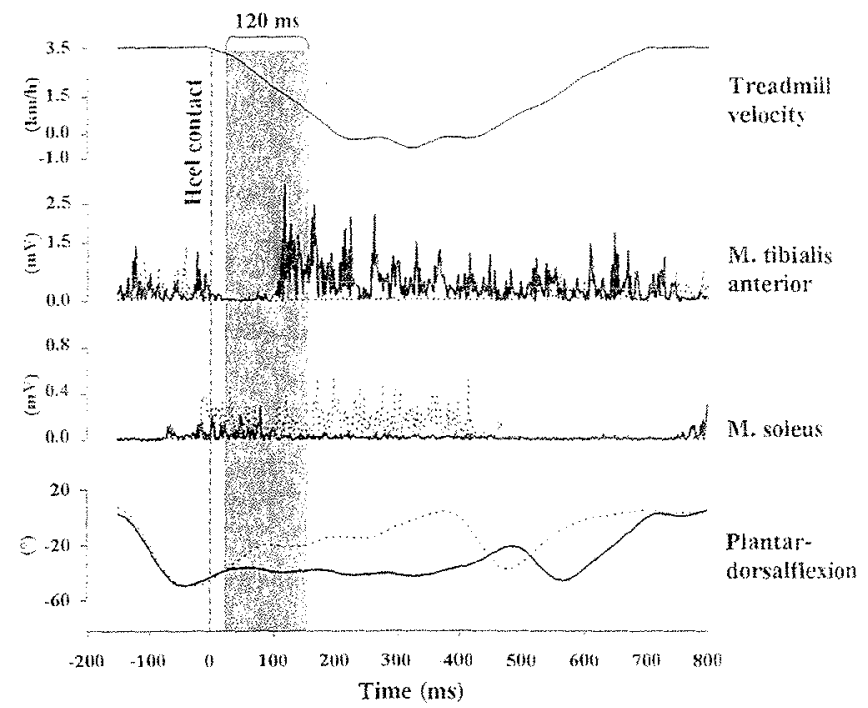

Fig. 1. Electronyographic (averaged and rectified) and goniometric data of the stance phase of a regular unperturbed gait cycle (dotted line) are synchronized at heel contact (dashed vertical line) with EMG and goniomeric data of a decelerating impuise / thick ine The analysed time interval was $120 \mathrm{~m}$ after the first response in the goniometer-signal to the perturbation impulse (functional reflex activiry: grey rectangle). The first part of the latency between heel strike and the onser of perturbation (beginning of the analyzed time interval) can be explained by mechanical delay due to the transmission and procession of the trigger signal coming from electrical switches implemented in the inlays of the righr shoe and the deceleration of the treadmill. Modified from Granacher et al. [8].

the foot) were used as a trigger for decelerating the treadmili precisely at hee contact and for determining the stance and the swing phase of a regular gait cycle. FRA was time analysed $120 \mathrm{~ms}$ after the first response to the perturbation impulse. More specifically, the first response to the perturbation impulse (trigger signal was indicated by a goniometer-signal (plantar/dorsalflexion of the ankle joint) (Fig. 1 ) Therefore, an electronic two-axial goniometer (Penny\&Giles". type XM180, Gwent UK) was firmly attached, with double-sided adhesive raps, along the Achilies tendon at the ankle joint. The goniometer-signal was fittered by a Butcerworth lowpass filter, using a cut-off frequency of $10 \mathrm{~Hz}$. With regard to the decelerating impulse, the inflection point of the goniometer-sigmal for plantar/dorsalflexion of the ankle was calculared and taken as a trigger for futher data analysis. Naxima anguiar velocity of the ankle joint was deremined in the time interval $120 \mathrm{~ms}$ following the first response in the goniometer-signal to the perturbation impulse (Fig. 1). Furthemore, latency defined as the time that elapses beween the first response in the goniomerer-signal and the first electromvoraphic (EMG) activity was analysed. Altogether, a total of 10 decelerating impuises on the treadmill were applied and averaged for further data analysis. Besides the perturbation situations the normai walking pattern (during unperturbed walking) was recorded at a speed of $3.5 \mathrm{~km} / \mathrm{h}$. Pinappels et al. [13] investigated EMG modulation in anicipation of a possible trip during walking in young and older adults. The authors reported that anticipatory effects did not jeopardize the validity of experiments in which subjects were perturbed more than once. Further, intraclass correlation coeficients (ICCs were calculated for FRA in TA $(I C C=0.85)$, latency in TA $(I C C=0.92)$, and maxima angular velocity of the ankle joint $(\mathrm{ICC}=0.88$ ) indicating excellent test-recest reliability. This protocol has recently been described in detail elsewhere $[8,9,14]$.

\subsection{Isokinetic fatigue protocol}

Right after the intial perturbation impulses on the treadmill, the participant right foot was positioned and frmly fixed in an isoknetic device (cybex: Orthodion, Medway. USA) while the subject was lving on a massage table in prone position with the waist fixed. Before the protocol started, subjects became accustomed to the isokinetic device by doing a warm-up consisting of five submaximal dynamic actions in a concentric-concentric mode at an angulat velocity of $60 \% \mathrm{~s}$ (plantar-dorsiflexion). Thereafter, each subjec: performed fout maximal contractions of the plantar. and dorsiflexors at $60 \% \mathrm{~s}$. For each trial. subjects were thoroughly instructed ro act as forcefully as possible. The best trial was taken as maximal coroue $\left\{F_{\max }\right\}$. The fatigue criteria were determined by examining the subjects $F_{\max }$ during each exercise No limitations were placed on the number of repetitions to reach $50 \%$ of $F_{\text {max }}$. Once three consecutive repeticions below $50 \% F_{m a x}$ were obtained the subject was inmediately unfixed from the isokinetic device and led to the treadmill were the decelerating perturbation impulses were applied. During the fatigue protocol, subjects were instructed to avoid forced respiration during maximal efforts. 
2.2.3. Electromyography (EMG)

Circular bipolar surace electrodes (Hellige". type $44008347 \mathrm{Ag} / \mathrm{AgCl}$. Freiburg, Germany) (diamerer $10 \mathrm{~mm}$, center to center distance $25 \mathrm{~mm}$ were placed over $\mathrm{m}$ tibialis anterior(TA), $m$. soleus ( 50 ), m. peroneus ( $P E$ ) and $m$. vastus medialis (VM) of the right leg. The longtudinal axes of the electrodes were in line with the direction of the underiying muscle fibers. The reference electrode was attached to the patel la listerelectrode resistance was kept below $5 \mathrm{k}$. by shaving, sightily roughening degreasing and dismfecting the skin. ENC signals were sampled at $500 \mathrm{~Hz}$, amplined and bandpass filtered (10-1000 Hz) and were carefuly monitored for artifacts, noise and cross-taik. ENG data were cuantified by integraring and time nomalising the full-wave rectified EMG signals (mean amplitude voltage (MAV) over the time interval of $120 \mathrm{~ms}$. in addition, standardized reference contractions (muscle activity during 10 averaged regular and unperturbed gair cycles) were used to normalise MAV of TA. SO and VM during the decelerating impulse (functional refiex activity) Thus. FRA during 10 decelerating impulses was expressed in per cent of muscle activity curing 10 regular gaic cycles. Antagonist coactivacion was expressed for gat perturbations as the ratio berween nomalized MAV of 50 and normalized MAV of TA, measured duning the same period of time.

\subsection{Statistical analysis}

Data are presented as group mean values $\div$ standard deviacions (SD). A multivariate analysis of variance (MANOVA) was used to detect differences between study groups (young, elderly) in all baseline variabies. In case of significant baseine differences, deltas (post-fatigue value-pre-fatigue value) were calculated for the respecting parameters and differences between young and elderly men were computed by a muitivariate ANOVA. If baseline differences between young and eiderly men were not present, parameters of the gait pertubation tests were analysed in separate 2 (groups: young, elderly) $\times 2$ (tests: pre-fatigue, post-fatigue) ANOVA with repeated measures on test, in addition, the classification of effect sizes was determined by calculating partial eta square $\left(n_{i}^{2}\right)$. The significance level was set at $p<.05$. All analyses were performed using Statistical Package for Social Sciences (SPSS version 16.0 .

\section{Results}

Table 2 displays means and standard deviations for all variables. At baseline, the elderly men showed a significantly lower normalized FRA in TA during gait perturbations compared to the young men $(p=.043)$. For all other collected variables, no significant differences were detected at baseline between young and elderly subjects. Further, the number of repetitions to reach $50 \%$ of $F_{\text {max }}$ was not significantly different between young and elderly men.

After the fatigue protocol, both groups (young, elderly) showed slightly longer latencies in $\mathrm{TA}$ in response to the decelerating perturbation impulse (Table 2). However, the analysis failed to indicate statistically significant main and interaction effects.

For both groups, a significant pre to post-test decrease in normalized FRA in TA (Fig. $2 ; p<.001$ ) was observed coming along with an increase in antagonist coactivity (Fig. 3; $p=.013$ ). However, no significant group $x$ test interaction was found for either parameter. The analysis of normalized activity in SO, PE, and VM during the compensation of gait perturbations failed to indicate significant main and interaction effects.

There was a significant pre to post-test increase in maximal angular velocity (plantar-dorsiflexion) of the ankle joint for both groups $(p=.007$; Fig. 4$)$, but no group $\times$ test interaction $(p=.374)$.

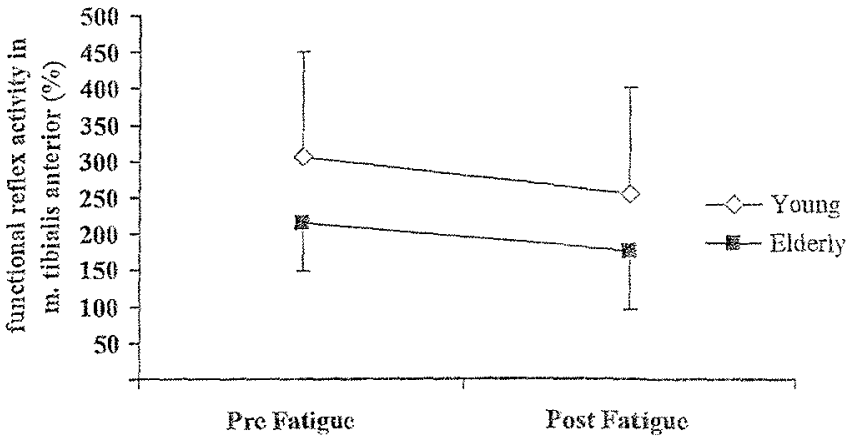

Fig. 2. Functional refiex activicy of $m$. thiblis anterior analysed in the time interval $0-120 \mathrm{~ms}$ and nomalized on muscle activity of the m. tibials anterior durng tize swing phase of 10 regular gat cycles, expressed in 8 are presented pre and posifatigue as mean $=$ standard deviation.

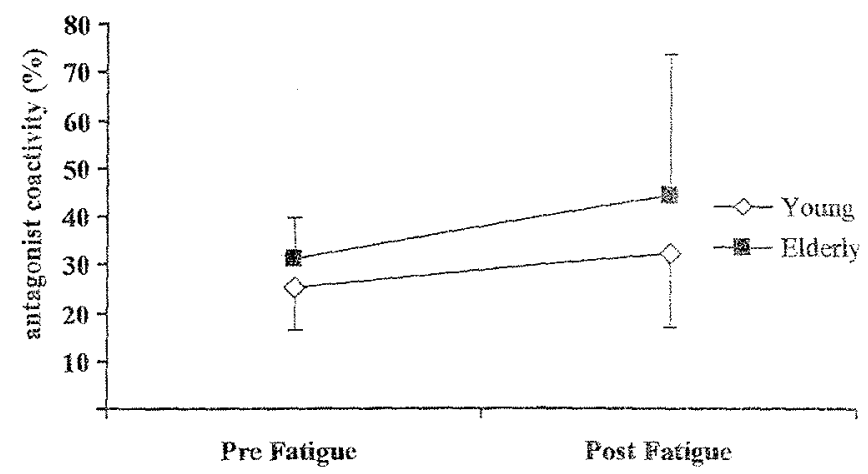

Fig. 3. Antagonist coactivity (m. soleus and $m$ tibialis anterior analysed in the the interval $0-120 \mathrm{~ms}$ and normalized on muscle activity of the m. tibiais anterior duning the swing phase and the $\mathrm{m}$. soleus during the stance phase of 10 regular gait cycles, expressed in $\%$ are presented pre and post-fatigue as mean sustandar deviation.

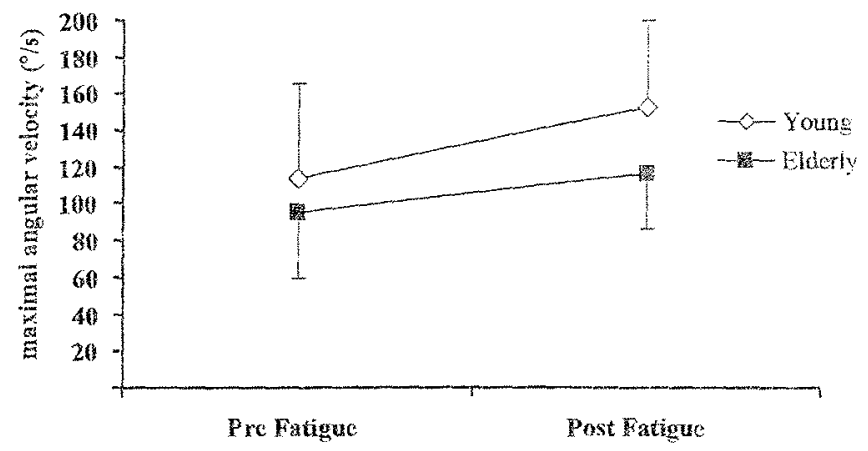

Fig. 4. Maximal angular velociry in the ankle joint (plantar-corsinexion analysed in the time interval $0-120 \mathrm{~ms}$, expressed in $/ \mathrm{s}$ is presented pre and post-fatigue as mean $=$ standard deviation.

Table 2

Effects of ankle fatigue on functional reflex accivity during gait perturbations in young and elderiy men.

\begin{tabular}{|c|c|c|c|c|}
\hline \multirow[t]{2}{*}{ Parameters } & \multicolumn{2}{|l|}{ Young men } & \multicolumn{2}{|l|}{ Elcerly men } \\
\hline & Pre-fatigue & Post-fatigue & Pre-fatigue & Post-fatigue \\
\hline Latency in m. tibialis anterior (ms) & $75.1 \leq 9.0$ & $79.6 \div 17.2$ & $77.7 \pm 13.0$ & $79.4=11.6$ \\
\hline FRA in $m$. tibialis anterior $(\%)$ & $306.1=144.9$ & $253.7=146.2$ & $215.7=66.4$ & $17.4 .7 \pm 79.7$ \\
\hline Activity in m. soleus $(\%)$ & $67.8=20.5$ & $7.3=27.6$ & $63.7 \pm 11.6$ & $68.9 \pm 31.0$ \\
\hline Coactivity (SO/TA) $(\%)$ & $25.1=8.7$ & $32.3 \pm 15.2$ & $31.3 \pm 8.4$ & $44.1 \div 29.6$ \\
\hline Acrivity in $\pi$. peroneus $(\%)$ & $148.9 \div 59.2$ & $141.1 \pm 82.4$ & $111.3 \pm 39.9$ & $115.1=51.8$ \\
\hline Activity in $m$. vastus medialis (\%) & $189.2 \div 134.1$ & $182.9 \div 93.0$ & $167.4 \div 49.3$ & $175.3=62.0$ \\
\hline Maximal angular velocity (/s) & $113.3 \div 51.9$ & $152.7=46.9$ & $95.6 \pm 35.3$ & $116.5=30.2$ \\
\hline
\end{tabular}

Notes: Values are mean $\div$ DD. FRA functional reflex activity during gair perturbations; $50=$ m. soleus: TA $=$ m. tibialis anterior. 


\section{Discussion}

From a functional point of view, the most interesting findings of the present study were that fatigue of the muscles encompassing the ankie joint had an impact on the ability to compensate for gait perturbations in both young and elderly men in terms of a fatigueinduced decrease in FRA of TA accompanied by an increase in coactivity of SO and TA. However, no significant differences between young and elderly subjects were detected regarding the impact of muscle fatigue on the ability to compensate for gait perturbations.

In the present study, elderly men showed smaller magnitudes of FRA in TA (prime mover) than young men when postural control was threarened by gait perturbations. The impaired ability of the elderly men to compensate for gait perturbations complies with literature [16,15]. Tang and Woollacott [15] found smaller magnitudes of postural responses and higher levels of antagonist coactivation to unexpected slips during walking in older adults. Lin and Woollacott [16] determined postural muscle response characteristics following various sizes of support surface perturbations in young, stable older, and unstable older adults. Slower onset latencies, smaller magnitudes of postural responses, and longer maintenance of postural muscle activation were found in response to platform perturbations in both stable and unstable older subjects compared to young adults.

It has been speculated that age-related changes in reflex activity are attributed to a desensitization of muscle spindles, a demyelination of sensory axons, with a subsequent decrease in number of sensory neurons, changes in presynaptic inhibition, and a reduction in number of inter- as well as alpha- and gamma-motoneurons $\mid 17\}$. In particular, the reduced afferent input via muscle spindles in old age could affect the control of the muscle's length and velocity of contraction and therefore the individual's ability to adequately respond to balance threats [18].

In the present study, fatigue of the muscles encompassing the ankle joint resulted in a decrease in FRA of TA during gait perturbations in both experimental groups. Furthermore, increases in coactivity and mean angular velocity in the ankle joint complex were observed in young and elderly men. These results suggest that fatigue may impair an indivicual's ability to correct for an unexpected decelerating perturbation impulse while walking on a treadmill. Our observations are in line with similar findings of previous investigators [19]. Jackson et al. [19] investigated the effects of fatigue and habituation on the stretch refiex of the ankle musculature in response to rapid inversion perturbations in a cohort of young healthy female and male adults. post-fatigue, the authors observed a significant decrease in reflex amplitude of the $\mathrm{m}$. peroneus longus and brevis and concluded that the diminished reflex amplitude may suggest reduced dynamic stability after fatigue. In a recent study including young and older adults, Mademli et al. (7) examined the control of dynamic stability after inducing a sudden perturbation from a fixed forward inclined position before and after submaximal fatiguing knee extension contractions. The authors reported that at baseline, the older adults showed a lower margin of stability at touchciown as well as at the end of the main stance phase. Postfatigue, no significant effects of fatigue were identified in both groups regarding the margin of stability after the forward falls. In contrast ro Mademli et al. [7], we found that fatigue of the muscles encompassing the ankle joint has an effect on dynamic stability in young and elderly men. This inconsistency may be due to the rather variable methodologies applied in the two studies. Mademii et al. [7] carried out their fatigue protocol on a leg extension weight machine. Subjects performed two-legged submaximal knee extension-flexion contractions at $25 \%$ of their maximum lifted weight until they could not lift the given weight through the whole range of motion anymore. According to Mademli et al. [7], strength of the knee extensors should be reduced by $20-25 \%$ after this protocol. In the present study, an isokinetic fatigue protocol at an angular velocity of $60 \% / \mathrm{s}$ was applied. Thus, mode of contraction, the fatigued muscle groups, as well as the intensity of the fatigue protocol differed substantially in the two studies. It seems likely that the intensity of the farigue protocol in the study of Mademli et al. (7) was too low to induce changes in balance recovery mechanics due to fatigue. In addition, Mademil et al: [7] examined balance recovery mechanics during forward falls from a biomechanic perspecrive by means of kinematic and dynamographic methods, whereas we investigated decelerating perturbation impulses from a neuromuscular perspective by means of electromyographic and goniometric methods.

In the present study, ankle fatigue diminished FRA in TA in young and elderly men. There is preliminary evidence that reflex responses contribute to joint stiffness and thus joint stability [20]. Thus, it could be argued that the observed decreases in FRA reduced joint stiffness and impaired joint stability. This seems to be supported by the fatigue-induced increase in maximal angular velocity of the ankle joint complex during the compensation of gait perturbations in young and elderly men in the current study. Thus, it can be speculated that as a result of fatigue and in an effort to compensate for facigue, our subjects increased coactivation of the TA (agonist) and the SO (antagonist) to enhance joint stiftness and consequently joint stability. This complies with literature, since other researchers have also found increases in agonist/antagonist coactivation following fatigue [21]. Furthermore, an association between alterations in coactivation and changes in ankle stifiness have been established $[20\}$.

The observed decrease in FRA of TA following fatigue could stem from small afferents of groups III and IV reacting to the products of muscle metabolism [22]. From animal studies, it is known that these afferents project on inhibicory interneurones [23], reduce the discharge rates of gamma motoneurones and modify Renshaw inhibition [24]. Other researchers propose that the dominant inhibitory effect from group-II and $V$ afferents in fatigue involves a reduction in excitatory input by presynaptic inhibition of the group la afferents [25]. However, it has been reported that the compensation of foot displacements during gait is most likely provided by polysynaptic spinal reflexes via group-11 muscle spindle afferents [26]. Thus, fatigue-induced presynaptic inhibition of the la terminals is unlikely to account for the observed decrease in FRA of TA during gait perturbations. Other mechanisms have to be considered. Since Rudomin and Schmidt [27] reported that presynaptic inhibition does not only affect primary afferents, but also group-1l afferents, it can be speculated that the metabolically induced activicy of the small myelinated and unmyelinated group-III and IV afferents causes presynaptic inhibition of group-ll afferents. Therefore, this mechanism might be responsible for the observed decreases in FRA of TA following fatigue. However, future studies have to elucidate this issue.

Our second hypothesis is still unresolved. Based on literature $[28,15]$, we speculated that the impaired ability to compensate for different perturbation impulses in old compared to young adults in an unfatigued condition can also be transferred to the fatigued condition. At baseline, we found significant differences berween young and elderly men regarding FRA in TA during gait perturbations. Post-fatigue, we could not detect these differences anymore. There is controversy in literature regarding the effect of muscle fatigue on voluntary muscle activation. McNeil and Rice [29] suggest that older adults exhibit greater fatigability during isotonic contractions than young adults. Other investigators have 
demonstrated similar fatigability in young and old subjects during isometric contractions [30], whereas still others have observed that older adults fatigue less than young during isometric contractions [31]. These inconsistencies may be due to the rather variable methodologies implemented to quantify fatigue. To the authors' knowledge, there is no other study avallable which invesigated the effects of fatigue of the muscles encompassing the ankle joint on FRA during gait perturbations in young and elderly subjects. Thus, comparable data are missing. However, based on the age-related motor unit remodeling theory [32]. it can be speculated that elderly subjects are more fatigue resistant than young subjects. In fact, it is reported that muscles containing a high percentage of type I fibers are more fatigue resistant and show higher reflex excitability than muscles containing high percentages of type 11 fibers [33]. This might explain why we did find differences at baseline, mainly caused by deficits in the proprioceptive system, but not post-fatigue.

Three potential limitations of this study warrant discussion. First, one might argue that isokinetic fatigue protocols cannot simulate functional fatigue that occurs during activities of daily living. However, the advantage of an isokinetic fatigue protocol lies in the high standard criteria level associated with this type of fatigue protocol. Furthermore, specific fatigue protocols for young and elderly adults could be applied in future studies to avoid misinterpretations of results [34]. Second, since there is contradictory information in literature regarding the impact of fatigue on reffex activity in males and females $[35,36]$, future studies should incorporate female subjects in a similar design. Third, the question remains unanswered regarding the time frame that is necessary to return the fatigue-induced deficits in dynamic postural control to baseline values. Therefore, future studies should involve additional post-fatigue testing times to elucidate this important issue. Despite the limitations presented, results of the study provide strong evidence that isokinetic fatigue impairs the ability to compensate for gait perturbations in young and elderly men.

In summary, the use of a muscle fatigue protocol with standardized fatigue criteria has been shown to decrease FRA in $T A$, to increase coactivation of muscles encompassing the ankle joint complex, and to increase maximal angular velocity in plantar-dorsiflexion direction of the ankle joint during gait perturbations in young and elderly men. However, no significant differences between young and elderly subjects were detected regarding the impact of muscle fatigue on the ability to compensate for gait perturbations. These results may imply that age-related deteriorations in the postural control system do not specifically affect the ability to compensate for gait perturbations under fatigued condition.

\section{Funding}

This work was supported by LGFG Grant (Landesgraduiertenförderung, Baden-Württemberg, Germany. Our sponsor did not have any influence on the design, methods, subject recruitment, data collections, analysis and preparation of this paper. The endorsement involved a scholarship (monthly salary) for the corresponding author from a state program.

\section{Confict of interest statement}

None of the authors have any conflicts of interest.

\section{Acknowledgment}

The authors would like to thank Dr. Phil. Harald Seelig for his statistical advice.

\section{References}

[1] Blake Al, Morgan K. Bendall M], Dallosso H, Ebrahim SE, Arie TH, et al. Falls by elderly people at home: prevalence and associated factors. Age Ageing 1988:17:365-72.

(2) Lord SR. Ward IA, Williams P. Anstey K. An epidemiological study of fals in older communiry-dwelling women: the Randwick falls and fractures study. Aust J Public Health 1993:17:240-5.

13) Rubenstein $\mathrm{L} Z$. Falls in older people: epidemiology, risk factors and surategies for prevention. Age Ageing 2006;35(Suppl. 2):i37-41

[4] Maki BE, Mcliroy WE. Control of rapid jimb movements for balance recovery: age-related changes and implications for fall prevention. Age Ageing 2006: 35 (5uppl. 2$): 1112-8$.

15] Helbostad IL. Leirfall S. Moe-Nilssen R. Sletvold O. Physical fatigue affects Eait characteristics in oldier persons. J Gerontol A Biol Sci Med $5 \mathrm{ci} 2007,62: 1010-5$

161 Pariat P. Lockhart TE. Effects of lower extremiry muscle facigue on the outcomes of slip-induced falls. Ergonomics 2008;51:1873-84.

17) Mademil L. Arampatzis A, Karamanidis K. Dynamic stability control in forward falis: postural corrections after muscle fatigue in young and older adults. Eur. Appl Physiol 2008:103:295-306.

(8) Granacher U Gollhofer A Strass D. Training induced adaptations in characteristics of postural reflexes in elderly men. Gait Posture 2006:24:459-66

(9) Granacher U, Gruber M, Gollhofer A. Force production capacicy and functional reflex acivity in young and elderly men. Aging Clin Exp Res 2009 [Epub ahead of print).

(10) Frey I Berg A. Grathwohl D. Keul I. Freiburg Questionatie of physical activitydevelopment, evaluarion and application. Soz Praventivmed 1999:44:55-64.

[11) Coren S. The lateral preference inventory for measurement of handedness. footedness, evedness, and earedness: noms for young adults. Bull Psychol soo $1993: 31: 1-3$.

(12) Gollhofer A, Schmidtbieicher D. Quintern ], Dietz V. Compensarory movemenrs following gait perturbations: changes in cinematic and muscular activation patems. In I Sports Med 1986:7:325-9.

(13) Pijnappels M. Bobbert MF, van Dieen JH. EMG modulation in anticipation of a possibje trip during walking in young and older aduits. Electromyogr Kinesiol $2006: 16: 137-43$

[14) Granacher U, Gruber $M$, Gollhofer $A$. The impact of sensorimotor training on postural control in elderiy men. Deut $Z$ Spormed 2009:60:387-93.

[15] Tang PF, Wooliacott MH. Ineficient postural responses to unexpected slips during walking in older adults. J Gerontol A Biol Sci Med Sci 1998;53:MA7180.

(16) Lin Si. Woollacott MH. Postural muscle responses foliowing changing balance threats in young, stable older, and unstable older aduits. I Mot Behav $2002: 34: 37-44$

(17) Granacher U, Zahner $L$, Golthofer A. Strength, power, and postural control in seniors: considerations for functional adaptations and for fali prevention. Eur Sport Sci 2008:8:325-40.

(18) Mynark RG, Koceja DM. Effects of age on the spina! stretch rellex I App Biomech 2001:17:188-203

(19] Jackson ND, Gutierfez GM Kaminski T. The effect of fatroue and habiluation on the stretch reflex of the ankje musculature. ] Electromyogr Kinesiol 2009 $19: 75-84$

[20] Granata KP, Wiison SE, Massimini AK, Gabriel R. Acrive sriffness of the ankie in response to inertial and elastic loads. J Electromyogr kinesiol 2004:34:599609 .

[21] Patikas D, Michailidis C, Bassa H, Kotzamanidis C. Tokmakidis S. Alexidu S, et at Electromyographic changes of agonist and anragonist calf muscies durmg maximum isometric induced fatigue. Int I Sports Ned 2002;2:285-9.

(22) Wense S. Stahnike M. Responses in muscle afferent fibres of sow conduction velocicy to contractions and ischaemia in the cat I Physiol $1983 \cdot 342 \cdot 383-97$

(23) Cleland CL. Rymer WZ. Erwards FR. Force-sensitive intemeurons in the spinai cord of the cat. Science 1982;217:652-5.

124) Ellaway PH. Nurphy PR. Tripathi A. Closely coupled excitation of gammamotoneurones by group 31 muscle afferents with low mechanical threshold in the cat. I Physio! 1982:331:481-98.

[25] Hunter SK. Duchareau], Enoka RM. Muscle fatigue and the mechanisms of task failure. Exerc Sport Sci Rev $2004: 32: 44-9$

[26] Grey M], Ladouceur M, Andersen IB, Nielsen JB. Sinkjaer T. Group II muscle afferents probably contribute to the medium latency soleus strerch refiex during walking in humans. I Physiol 2001:534:925-33

[27] Kudomin P. Schmidt RF. Presynaptic inhibition in the vertebrate spinal cord revisited. Exp Brain Res 1999:129:1-37.

28) Pavol Ml. Runtz EF. Edwards B]. Pai YC. Age influences the outcome of a slipping perturbacion during initial but not repeated exposures, I Gerontol $A$ Biol Sci Med Sci 2002:57:M496-503.

(29) MoNeil C]. Rice CL. Fatigabihy is increased with age during velocity-dependent contractions of the dorsflexors. J Gerontol A Biol Sc Med Scl 200 ? $62: 624-9$.

30| Allman BL, Rice CL. incomplete recovery of voluntary isometric force after fatigue is not affected by old age. Muscie Nerve 2001,24:1156-67.

131] Kent-Braun IA. Ng AV. Doyle JW, Towse TF. Human skeletal muscle responses vary with age and gender during fatigue due to incremental isometric exercise. J Appl Plyysiol 2002:93:1813-23.

[32] Connelly DM, Rice $C L$, Roos MR, Vandervoot AA, Motor unit firing rates and contractile properties in tibialis anterior of young and old men. I Appl Physiol $1999 ; 87: 843-52$ 
133] Anderson ], Almeida-Siveira MI, Perot C. Reflex and nuscular adaptations in rat soleus muscle afrer hindlimb suspension. ] Exp Biol 1999:202:2701-7.

(34) Yoon T. De Lap BS, Grifith EE, Huncer SK. Age-related muscle fatigue after low-fore fatiguing contraction is explained by central fatigue. Muscle Nerve $2008: 37: 457-66$
[35] Moore BD, Droun], Gansneder BM, Shulz S]. The differential effects of fatigue on reflex response timing and amplitude in males and females. J Electromyag Kinesiol 2002;12:351 -60.

(36) Wilson EL. Madigan ML. Effects of fatigue and gender on peroned reflexes elicited by sudden ankle inversion. I Electromyogr Kinesiol 2007:17:160-6. 